\title{
环形腔激光器构成的激光网络对信号的同步比例 传输
}

\author{
吕翎"，李成仁，李钢，田静，顾佳佳，王周洋 \\ 辽宁师范大学物理与电子技术学院, 大连 116029 \\ *联系人, E-mail: luling1960@aliyun.com
}

收稿日期: 2015-02-28; 接受日期: 2015-03-23; 网络出版日期: 2015-05-04

辽宁省理论物理重点学科项目 (编号: 905061)和辽宁省教育厅科学技术项目(编号: L2013410)资助

\begin{abstract}
摘要基于稳定性理论设计了一种同步比例传输技术, 用以实现具有时空混沌行为的激光网络对目标 信号的同步比例传输. 通过选择特定的 Lyapunov 函数，确定了网络节点控制输入的结构. 同步过程中， 目标信号允许在网络的任意一个节点输入. 运用此项技术不但能够使激光网络按照比例传输因子的大小 同步传输目标信号，而且网络的连接方式和节点数目不受任何条件的约束. 仿真结果显示，网络的同步 性能非常稳定.
\end{abstract}

关键词同步比例传输, 复杂网络, 时空混沌, 环形腔激光器

PACS: 05.45.Xt, 05.45.Pq, 05.45.Ra

doi: 10.1360/SSPMA2015-00087

\section{1 引言}

具有横向效应的环形腔激光器的激光输出, 在 光通信、信息编码、光纤传感等许多方面展示了诱人 的应用前景. 理论和实验研究均表明, 横向效应对激 光器的动力学行为起着至关重要的作用. 特别是在 适当的增益值下, 环形腔激光器的激光输出能够呈 现时空混沌行为 ${ }^{[1]}$. 近年来的研究使人们进一步发现, 利用诸如环形腔激光器这样的时空混沌系统构成激 光网络在大幅度提高其输出功率、远程通信以及输出 信号的同步转换与传输等方面起着得天独厚的作用, 尤其是输出信号的同步转换与传输其应用潜力广泛, 因此, 激光网络同步的相关研究已成为迄今国内外
研究的新热点.

网络同步的理论研究源于 Pecora 和 Carroll 的标 志性工作 ${ }^{[2]}$. 在文献[2]中, Pecora 和 Carroll 给出了连 续时间耦合网络的具体表示以及网络完全同步的定 义. 进一步提出了主稳定函数判据并基于这一判据 推出了网络完全同步的条件. 在此之后, Gade 和 $\mathrm{Hu}^{[3]}$ 将这种网络同步方法的研究对象从规则网络推广到 了小世界网络. 鉴于实际网络节点之间信号的传输 和响应具有时滞效应, Earl 和 Strogatz ${ }^{[4]}$ 提出了一种实 现时滞网络的同步方法, 给出了时滞网络的同步判 据. 在实际应用中, 人们往往希望网络的连接方式可 以随时间的推移而变化, 为此, Lü 等人 ${ }^{[5,6]}$ 构造了一

引用格式: 吕领, 李成仁, 李钢, 等. 环形腔激光器构成的激光网络对信号的同步比例传输. 中国科学: 物理学 力学 天文学, 2015, 45: 060501 Lü L, Li C R, Li G, et al. Signal proportion transmission with a network constituted by lasers with ring cavities (in Chinese). Sci Sin-Phys Mech Astron, 2015, 45: 060501, doi: 10.1360/SSPMA2015-00087 
类时变动态网络模型, 并研究了这种时变动态网络 模型的同步特性. $\mathrm{Li}$, Chen 和 $\mathrm{Ji}$ 等人 ${ }^{[7-9]}$ 也相继对线性 或非线性耦合时变动态网络的同步问题进行了进一 步的研究.

近年来, 人们对复杂网络同步的研究更加系统 广泛 ${ }^{[10-16]}$. 人们围绕各种网络同步以及提高网络的 同步化能力等问题进行了深入的研究, 提出了许多 卓有成效的同步方法, 其中典型的有主稳定函数 法 ${ }^{[2]}$ 、自适应法 ${ }^{[17,18]}$ 、脉冲控制法 ${ }^{[19]}$ 以及牵制控制法 等 ${ }^{[20,21]}$. 并且, 网络同步的类型也不仅仅局限于网络 的完全同步, 还有网络的相同步 ${ }^{[22]}$ 以及广义同步 ${ }^{[23,24]}$ 之分. 特别是随着研究的不断深入, 人们已不限于研 究一个网络内部所有节点之间的同步规律, 还扩展 到网络与网络之间以及网络与外部信号之间的同步 研究 ${ }^{[25-28]}$. 这些重要的研究成果为进一步进行网络 同步的理论研究奠定了良好的工作基础, 同时也增 加了实际应用的可行性.

然而, 由于网络本身存在大量的具有相互关联 的节点, 处理起来非常繁琐, 使得至今网络同步方法 还相对较少, 且受到许多局限. 例如, 主稳定函数法 要求网络中所有节点以及节点之间的耦合函数必须 是同结构的, 并且同步流形必须是不变流形. 另外还 要求节点的耦合方式能够使网络在同步流形附近线 性化. 这些局限往往导致网络同步方法的实用性欠 佳. 因此, 改善原有的网络同步技术以及提出新的实 用有效的网络同步方案是十分必要的.

本文设计了一种简便快速的同步比例传输技术, 用以实现具有时空混沌行为的激光网络对目标信号 的同步比例传输. 同步过程中, 目标信号允许在网络 的任意一个节点输入. 运用此项技术不但能够使激 光网络按照比例传输因子的大小同步传输目标信号, 而且网络的连接方式和节点数目可以任意选择.

\section{2 环形腔激光器模型以及激光网络}

考虑激光的横向效应后, 描述环形腔激光器的 Maxwell-Bloch 方程变得相对复杂. 主要因为此时存 在光场的有限横截面与光场振幅和位相的径向变化 引起的衍射效应、谐振腔球面镜引起的波形畸变以及 反转粒子数的横向和纵向分布引起的增益变化. 文 献[29]详细研究了考虑增益介质的环形腔激光器的 动力学行为, 得到如下一维迭代方程:

$$
I_{n+1}=I_{n} \exp \left(\frac{\alpha}{1+I_{n}}-\Gamma\right),
$$

其中状态变量 $I_{n}$ 为归一化的光场强度. 参量 $\alpha$ 和 $\Gamma$ 分 别为增益系数和腔损耗.

在此之后, Wang 等人 ${ }^{[30]}$ 研究了考虑光场的有限 横截面与光场振幅和位相的径向变化引起的衍射效 应且在慢变振幅条件下的光强方程

$$
\frac{\partial I}{\partial t}=F(I)+D \nabla^{2} I
$$

其中 $F(I)$ 为描述光强的非线性函数, $D$ 为空间衍射耦 合系数, $\nabla^{2}=\frac{\partial^{2}}{\partial x^{2}}+\frac{\partial^{2}}{\partial y^{2}}$.

进一步将(2)式离散化后, 可以得到考虑横向效 应后环形腔激光器模型的光强方程

$$
\begin{aligned}
I_{n+1}(i, j)= & (1-D) f\left(I_{n}(i, j)\right)+\frac{D}{4}\left[f\left(I_{n}(i-1, j)\right)\right. \\
& +f\left(I_{n}(i+1, j)\right)+f\left(I_{n}(i, j-1)\right) \\
& \left.+f\left(I_{n}(i, j+1)\right)\right],
\end{aligned}
$$

这里 $i$ 和 $j$ 是离散化的空间格点坐标, $n$ 为离散化的 时间. 局域函数 $f\left(I_{n}(i, j)\right)$ 由(1)式来描述.

我们利用环形腔激光器模型的最大 Lyapunov 指 数来描述其动力学特性. 取系统的空间尺寸为 $100 \times 100$, 参量 $D=0.2, \Gamma=11$, 数值计算方程(3)的最大 Lyapunov 指数随参量 $\alpha$ 的演化关系如图 1 所示.

由图 1 可以确定参量 $\alpha=13 \frac{4}{9}$ 是模型最大

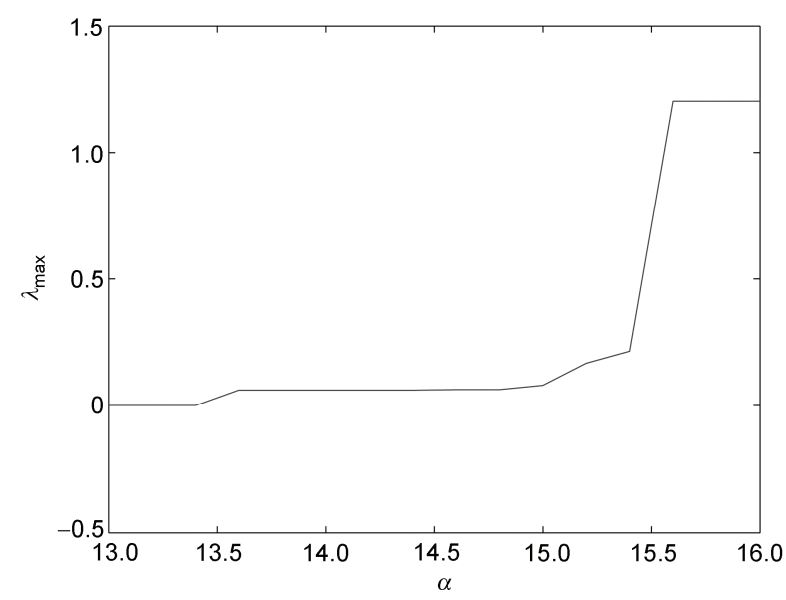

图 1 最大 Lyapunov 指数随参量 $\alpha$ 的演化关系

Figure 1 Evolution of maximum Lyapunov exponent with parameter $\alpha$. 
Lyapunov 指数正负转变的临界值. 当参量 $\alpha>13 \frac{4}{9}$ 后, 最大 Lyapunov 指数大于零, 环形腔激光器的输 出光强由原来的均匀输出过渡为时空混沌态, 即湍 流. 我们在最大 Lyapunov 指数大于零所对应的参量 区域内取 $\alpha=15$, 计算 $n=500$ 时环形腔激光器模型 的光强方程的相图、状态变量的时空演化及其投影斑 图如图 2-4 所示.

连接若干台环形腔激光器进行信号的比例传输. 在实际中这些激光器的连接方式应该是可以任意改 变的, 连接的激光器的数目也应该是任意的. 这些环 形腔激光器连接后就构成了一个激光网络. 网络的 各个节点就是单个的环形腔激光器, 网络的边就是

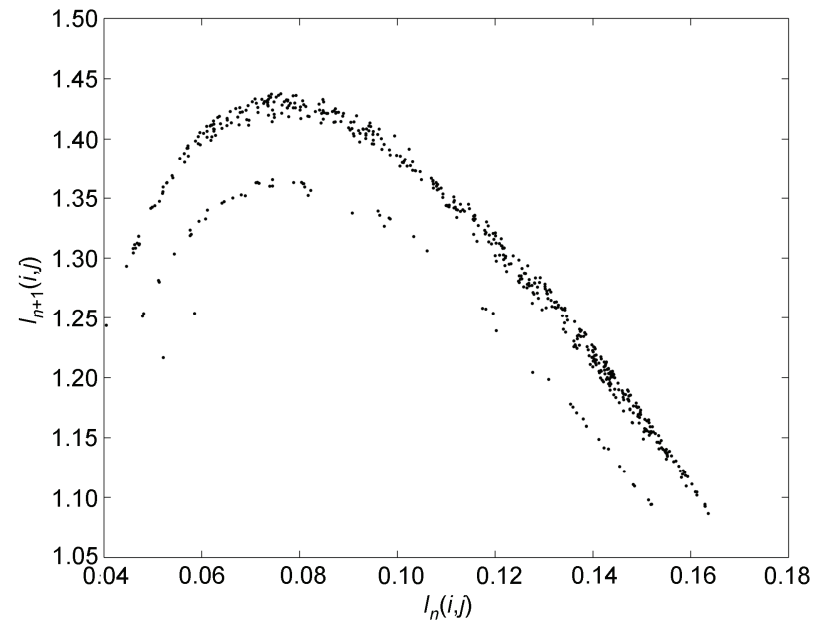

图 2 光强方程(3)的相图

Figure 2 Phase diagram of the light intensity eq. (3).

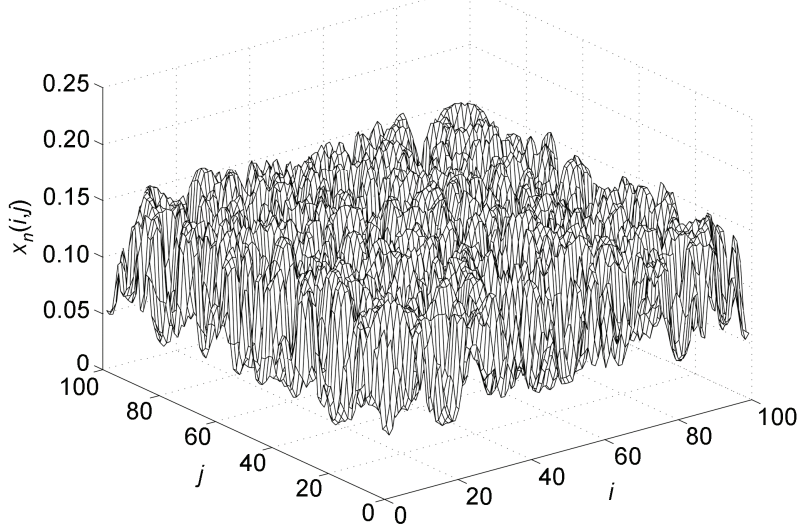

图 3 光强方程(3)状态变量的时空演化

Figure 3 Spatiotemporal evolution of state variable in the light intensity eq. (3).

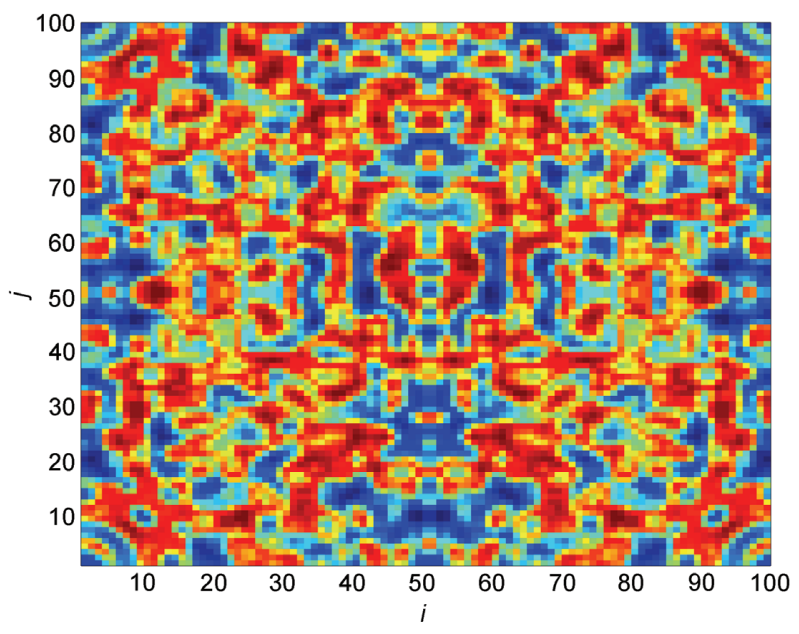

图 4 (网络版彩图)光强方程(3)状态变量时空演化的投影 斑图

Figure 4 (Color online) Projection pattern of spatiotemporal evolution of state variable in the light intensity eq. (3)

这些激光器之间的连接. 这样, 描述具有 $N$ 个节点的 网络方程为

$$
\begin{aligned}
& I_{n+1}^{k}(i, j)=\Sigma\left(I^{k}\right)+\delta_{k} \sum_{m=1}^{N} g_{k m} I_{n}^{k}(i, j)+u_{k}(i, j) \\
& (k=1,2, \cdots, N),
\end{aligned}
$$

这里 $\Sigma\left(I^{k}\right)$ 表示第 $k$ 个节点处的动力学方程, 即单个 环形腔激光器的光强方程(3)

$$
\begin{aligned}
\Sigma\left(I^{k}\right)= & (1-D) f\left(I_{n}^{k}(i, j)\right)+\frac{D}{4}\left[f\left(I_{n}^{k}(i-1, j)\right)\right. \\
& +f\left(I_{n}^{k}(i+1, j)\right)+f\left(I_{n}^{k}(i, j-1)\right) \\
& \left.+f\left(I_{n}^{k}(i, j+1)\right)\right],
\end{aligned}
$$

$\delta_{k} \sum_{m=1}^{N} g_{k m} I_{n}^{k}(i, j)$ 表示网络的边. 其中 $\delta_{k}$ 是网络节点之 间的耦合强度, $g_{k m}$ 是耦合矩阵元, 它决定网络的连接 方式与结构. 若网络中的两个节点之间存在连接, 则 $g_{k m}=1(k \neq m)$; 反之 $g_{k m}=0(k \neq m)$. 且 对角元 $g_{k k}=-\sum_{\substack{m=1 \\ m \neq k}}^{N} g_{k m} \cdot u_{k}(i, j)$ 是网络节点的控制输入.

假设网络传输的信号 $S_{n}$ 由单独一台环形腔激光 器发送

$$
S_{n}=I_{n}^{\mathrm{sign}}(i, j) .
$$

网络节点状态变量与目标信号之间的误差可以 写成 


$$
e_{n}^{k}(i, j)=I_{n}^{k}(i, j)-\beta_{k} I_{n}^{\mathrm{sign}}(i, j) \quad(k=1,2, \cdots, N),
$$

其中 $\beta_{k}$ 为比例传输因子.

定义 1 若存在 $\lim _{t \rightarrow \infty}\left|I_{n}^{k}(i, j)-\beta_{k} I_{n}^{\mathrm{sign}}(i, j)\right|=0$, 则 激光网络中的 $N$ 台环形腔激光器按照比例传输因子 的大小同步传输了目标信号.

\section{3 网络同步比例传输技术的设计}

设计一个正定的函数 $\left|\Psi_{n}^{k}(i, j)\right|$ 作为 Lyapunov 函 数, 且

$$
\Psi_{n}^{k}(i, j)=e_{n}^{k}(i, j)+\varphi_{n}^{k}(i, j),
$$

这里 $\varphi_{n}^{k}(i, j)$ 为待定函数. 我们设计它满足以下的递 推关系

$$
\varphi_{n}^{k}(i, j)=\varphi_{n-1}^{k}(i, j)+\mu_{k} e_{n-1}^{k}(i, j),
$$

式中的 $\mu_{k}$ 为配置参量.

依据稳定性理论, 设计的 Lyapunov 函数在零点 处须趋于零. 基于 (8) 和(9)式, 则有

$$
e_{n+1}^{k}(i, j)=\left(1-\mu_{k}\right) e_{n}^{k}(i, j),
$$

容易看出, 只要存在 $0<\mu_{k}<2$, 则网络节点与目标 信号之间的误差将渐进到零, 进而使设计的 Lyapunov 函数满足在零点处须趋于零的条件.

定理 1 当网络节点的控制输入为 $u_{k}(i, j)=\left(1-\mu_{k}\right)$ $e_{n}^{k}(i, j)-\Delta \Sigma\left(I^{k}, I^{\mathrm{sign}}\right)-\delta_{k} \sum_{m=1}^{N} g_{k m} I_{n}^{k}(i, j)+\xi_{k} \Psi_{n}^{k}(i, j)$, 且其 中的调节参量 $\xi_{k}$ 的取值范围为 $-2<\xi_{k}<0$ 时, 激光 网络中的 $N$ 台环形腔激光器能够按照比例传输因子 的大小同步传输目标信号.

证明：基于(7)式，可以进一步得到网络节点与 目标信号之间的误差演化方程

$$
\begin{aligned}
e_{n+1}^{k}(i, j) & =I_{n+1}^{k}(i, j)-\beta_{k} I_{n+1}^{\mathrm{sign}}(i, j) \\
& =\Delta \Sigma\left(I^{k}, I^{\mathrm{sign}}\right)+\delta_{k} \sum_{m=1}^{N} g_{k m} I_{n}^{k}(i, j)+u_{k}(i, j),
\end{aligned}
$$

这里 $\Delta \Sigma\left(I^{k}, I^{\mathrm{sign}}\right)=\Sigma\left(I^{k}\right)-\beta_{k} \Sigma\left(I^{\mathrm{sign}}\right)$.

基于 Lyapunov 定理，当 Lyapunov 函数满足

$$
\left|\Psi_{n+1}^{k}(i, j)\right|<\left|\Psi_{n}^{k}(i, j)\right|,
$$

则激光网络中的 $N$ 台环形腔激光器能够照比例传输 因子的大小同步传输目标信号.

容易看出, (12)式等价于下列两个不等式

$$
\begin{aligned}
& \Psi_{n}^{k}(i, j)\left[\Psi_{n+1}^{k}(i, j)-\Psi_{n}^{k}(i, j)\right]<0, \\
& \Psi_{n}^{k}(i, j)\left[\Psi_{n+1}^{k}(i, j)+\Psi_{n}^{k}(i, j)\right]>0 .
\end{aligned}
$$

这是因为基于(13)和(14)式，可以得到

$$
\left[\Psi_{n}^{k}(i, j)\right]^{2}\left\{\left[\Psi_{n+1}^{k}(i, j)\right]^{2}-\left[\Psi_{n}^{k}(i, j)\right]^{2}\right\}<0,
$$

由此得到

$$
\left[\Psi_{n+1}^{k}(i, j)\right]^{2}<\left[\Psi_{n}^{k}(i, j)\right]^{2} .
$$

显然，上述表示与(12)式一致.

设计网络节点的控制输入形式为

$$
\begin{aligned}
u_{k}(i, j)= & \left(1-\mu_{k}\right) e_{n}^{k}(i, j)-\Delta \Sigma\left(I^{k}, I^{\mathrm{sign}}\right) \\
& -\delta_{k} \sum_{m=1}^{N} g_{k m} I_{n}^{k}(i, j)+\xi_{k} \Psi_{n}^{k}(i, j),
\end{aligned}
$$

式中的 $\xi_{k}$ 为调节参量.

将(8)，(9)以及(11)式代入(13)式，并考虑到网络 节点的控制输入(17)式, 则可推出下列关系式

$$
\begin{aligned}
\Psi_{n}^{k}(i, j)\left[\Psi_{n+1}^{k}(i, j)-\Psi_{n}^{k}(i, j)\right] \\
=\Psi_{n}^{k}(i, j)\left[e_{n+1}^{k}(i, j)-\left(1-\mu_{k}\right) e_{n}^{k}(i, j)\right] \\
=\Psi_{n}^{k}(i, j)\left[\Delta \Sigma\left(I^{k}, I^{\mathrm{sign}}\right)+\delta_{k} \sum_{m=1}^{N} g_{k m} I_{n}^{k}(i, j)\right. \\
\left.\quad+u_{k}(i, j)-\left(1-\mu_{k}\right) e_{n}^{k}(i, j)\right] \\
=\Psi_{n}^{k}(i, j)\left[\xi_{k} \Psi_{n}^{k}(i, j)\right] \\
=\xi_{k}\left[\Psi_{n}^{k}(i, j)\right]^{2}<0,
\end{aligned}
$$

由此看出, 调节参量 $\xi_{k}$ 必须满足

$$
\xi_{k}<0 .
$$

同理，将(8)，(9)以及(11)式代入(14)式，并考虑 到网络节点的控制输入(17)式, 则可推出下列关系式

$$
\begin{aligned}
& \Psi_{n}^{k}(i, j)\left[\Psi_{n+1}^{k}(i, j)+\Psi_{n}^{k}(i, j)\right] \\
& =\Psi_{n}^{k}(i, j)\left[e_{n+1}^{k}(i, j)-\left(1-\mu_{k}\right) e_{n}^{k}(i, j)+2 \Psi_{n}^{k}(i, j)\right] \\
& =\Psi_{n}^{k}(i, j)\left[\Delta \Sigma\left(I^{k}, I^{\mathrm{sign}}\right)+\delta_{k} \sum_{m=1}^{N} g_{k m} I_{n}^{k}(i, j)+u_{k}(i, j)\right. \\
& \left.-\left(1-\mu_{k}\right) e_{n}^{k}(i, j)+2 \Psi_{n}^{k}(i, j)\right] \\
& =\Psi_{n}^{k}(i, j)\left[\left(\xi_{k}+2\right) \Psi_{n}^{k}(i, j)\right] \\
& =\left(\xi_{k}+2\right)\left[\Psi_{n}^{k}(i, j)\right]^{2}>0 .
\end{aligned}
$$

由此看出, 调节参量 $\xi_{k}$ 必须满足

$$
\xi_{k}>-2 \text {. }
$$

由于激光网络与目标信号之间实现同步比例传 输必须同时满足(19)和(21)式, 因此, 我们确定出调 
节参量 $\xi_{k}$ 的取值范围

$$
-2<\xi_{k}<0
$$

\section{4 仿真分析与讨论}

在这一部分, 我们将通过数值仿真来验证上述 网络同步比例传输技术的有效性.

由于设计的网络同步比例传输技术中, 激光网 络中节点的连接方式和节点数目可以是任意的, 所 以我们在数值仿真时任取激光器的台数并基于(4)式 构成激光网络, 且连接方式任取下列几种形式, 见图 5 .

基于上述网络连接(a)-(c), 分别写出相对应的单 向连接的耦合矩阵

$$
\begin{aligned}
G^{(\mathrm{a})}\left(g_{k m}\right) & =\left[\begin{array}{ccc}
-1 & 0 & 1 \\
1 & -1 & 0 \\
0 & 1 & -1
\end{array}\right], \\
G^{(\mathrm{b})}\left(g_{k m}\right) & =\left[\begin{array}{cccc}
0 & 0 & 0 & 0 \\
1 & -1 & 0 & 0 \\
1 & 0 & -1 & 0 \\
1 & 0 & 0 & -1
\end{array}\right],
\end{aligned}
$$

(a)

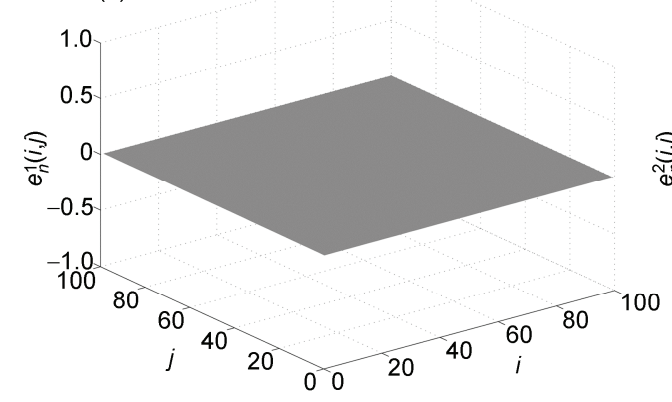

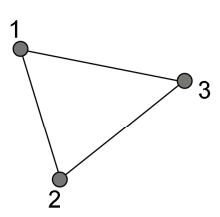

(a)

$$
G^{(\mathrm{c})}\left(g_{k m}\right)=\left[\begin{array}{ccccc}
0 & 0 & 0 & 0 & 0 \\
1 & -1 & 0 & 0 & 0 \\
0 & 1 & -1 & 0 & 0 \\
0 & 0 & 1 & -1 & 0 \\
0 & 1 & 0 & 1 & -2
\end{array}\right] .
$$

网络节点的控制输入由(17)式来确定, 配置参量 $\mu_{k}$ 和调节参量 $\xi_{k}$ 在满足约束 $0<\mu_{k}<2$ 以及 $-2<\xi_{k}<0$ 的 前提下, 任取 $\mu_{k}=0.8, \xi_{k}=-0.5$. 网络节点之间的耦合 强度取 $\delta_{k}=1$. 对应图 5(a)的网络连接, 比例传输因子 $\beta_{k}$ 任取为 $\beta_{1}=0.5, \beta_{2}=2, \beta_{3}=-0.5$. 仿真模拟发现, 在时 间演化 $n=80$ 左右网络开始按照比例传输因子的大小 同步传输目标信号, 并且在此之后一直保持稳定传 输. 我们给出 $n=500$ 时激光网络节点状态变量与目标 信号之间误差的时空演化以及网络节点与目标信号 的相图如图 6-7 所示. 通过图 6 可以清楚地看到, 激

图 5 激光网络的连接示意图

Figure 5 Connection diagrams of laser networks.

(b)

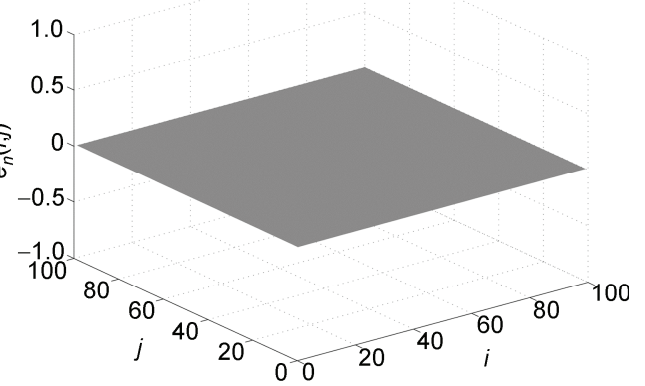

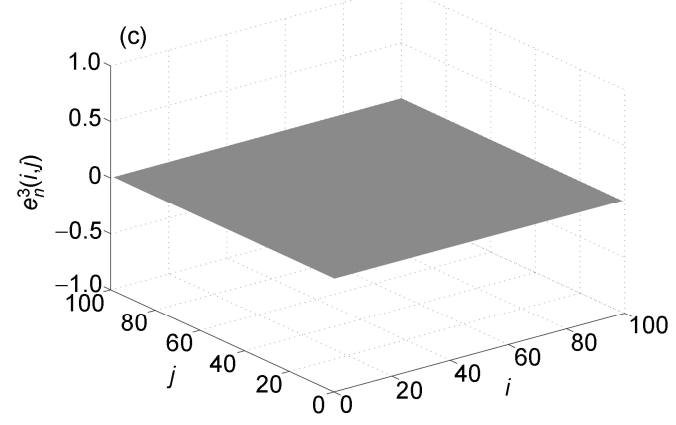

图 6 激光网络节点与目标信号之间误差的时空演化

Figure 6 Spatiotemporal evolutions of errors between the network node and the target signal. 

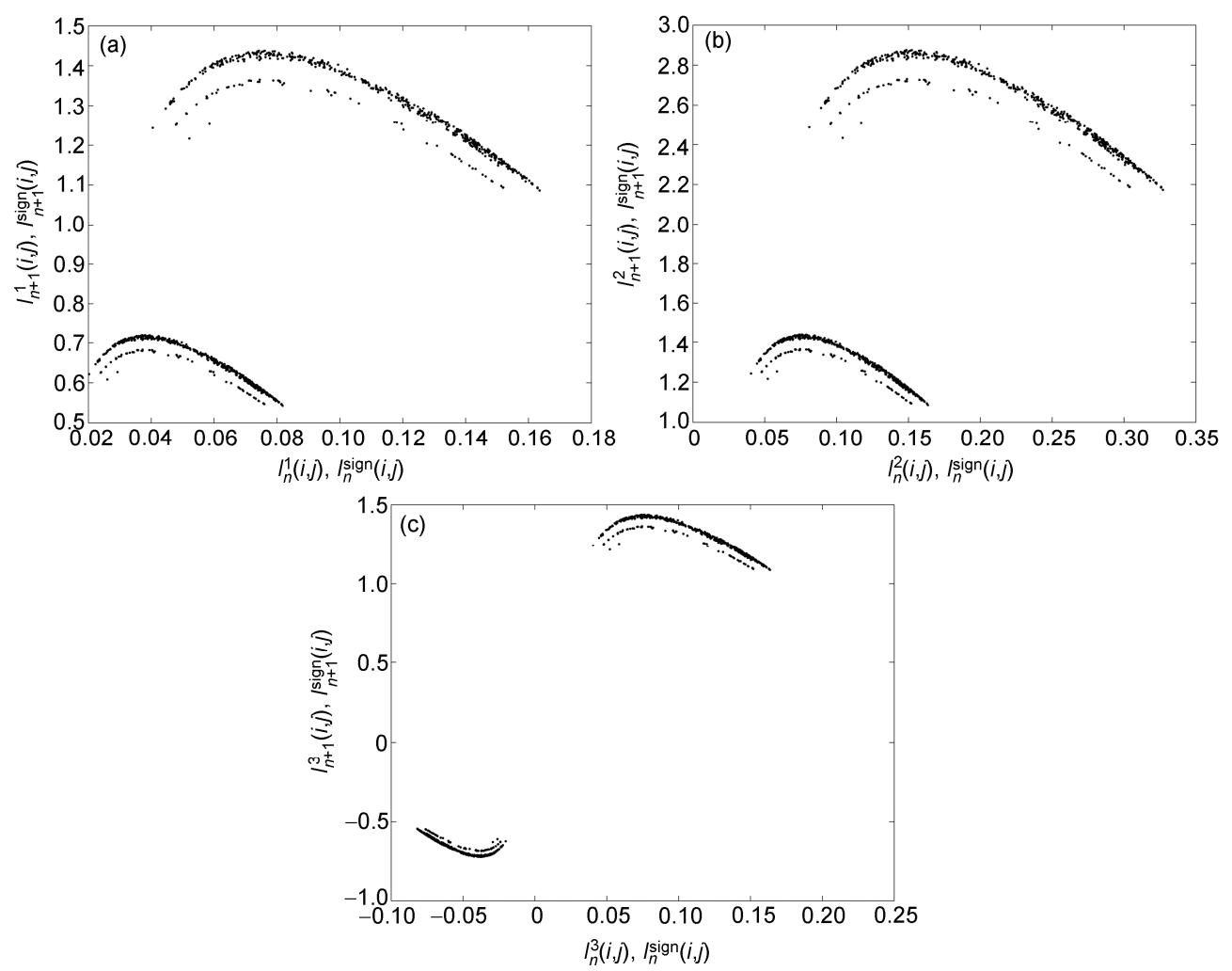

\section{图 7 网络节点与目标信号的相图}

Figure 7 Phase diagrams of the network node and the target signal.

(a)
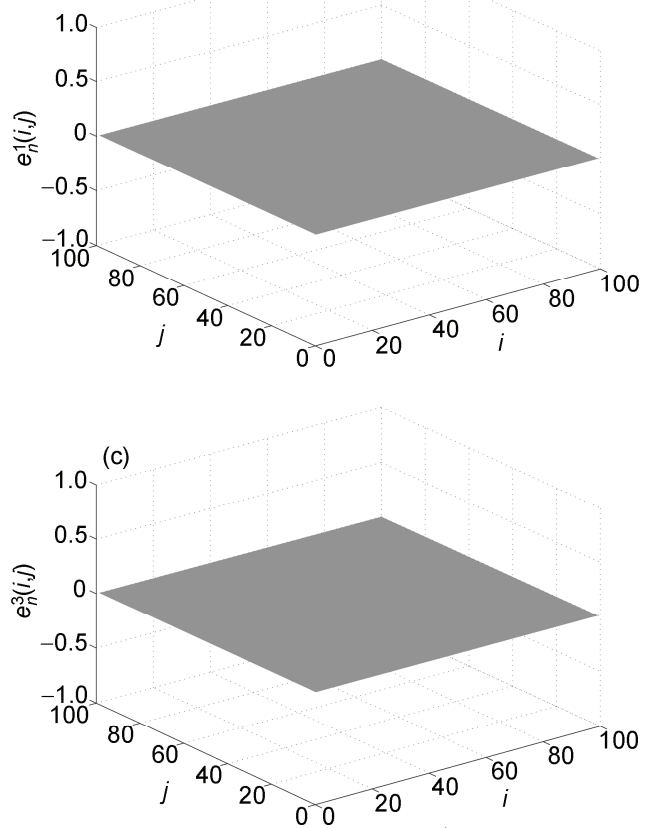

(b)
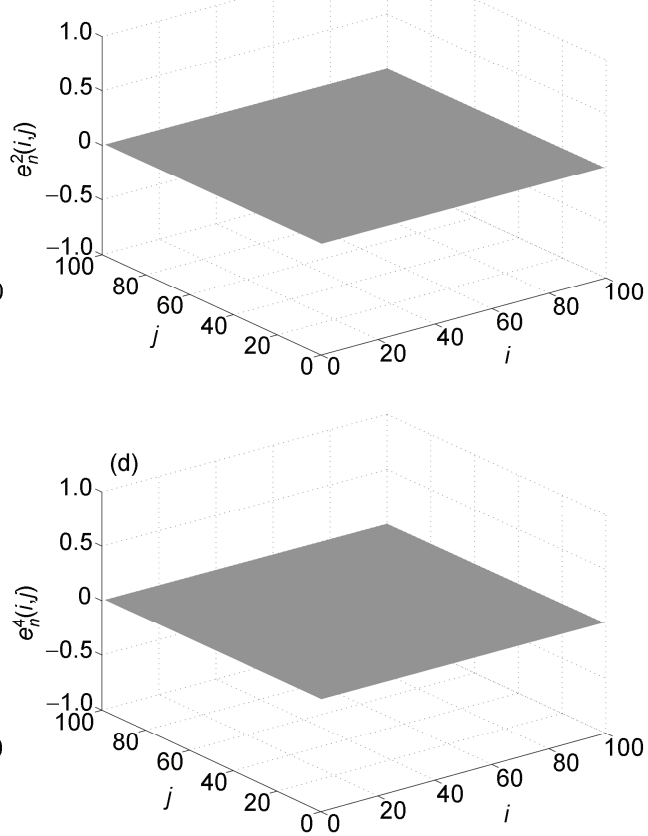

图 8 激光网络节点与目标信号之间误差的时空演化

Figure 8 Spatiotemporal evolutions of errors between the network node and the target signal. 

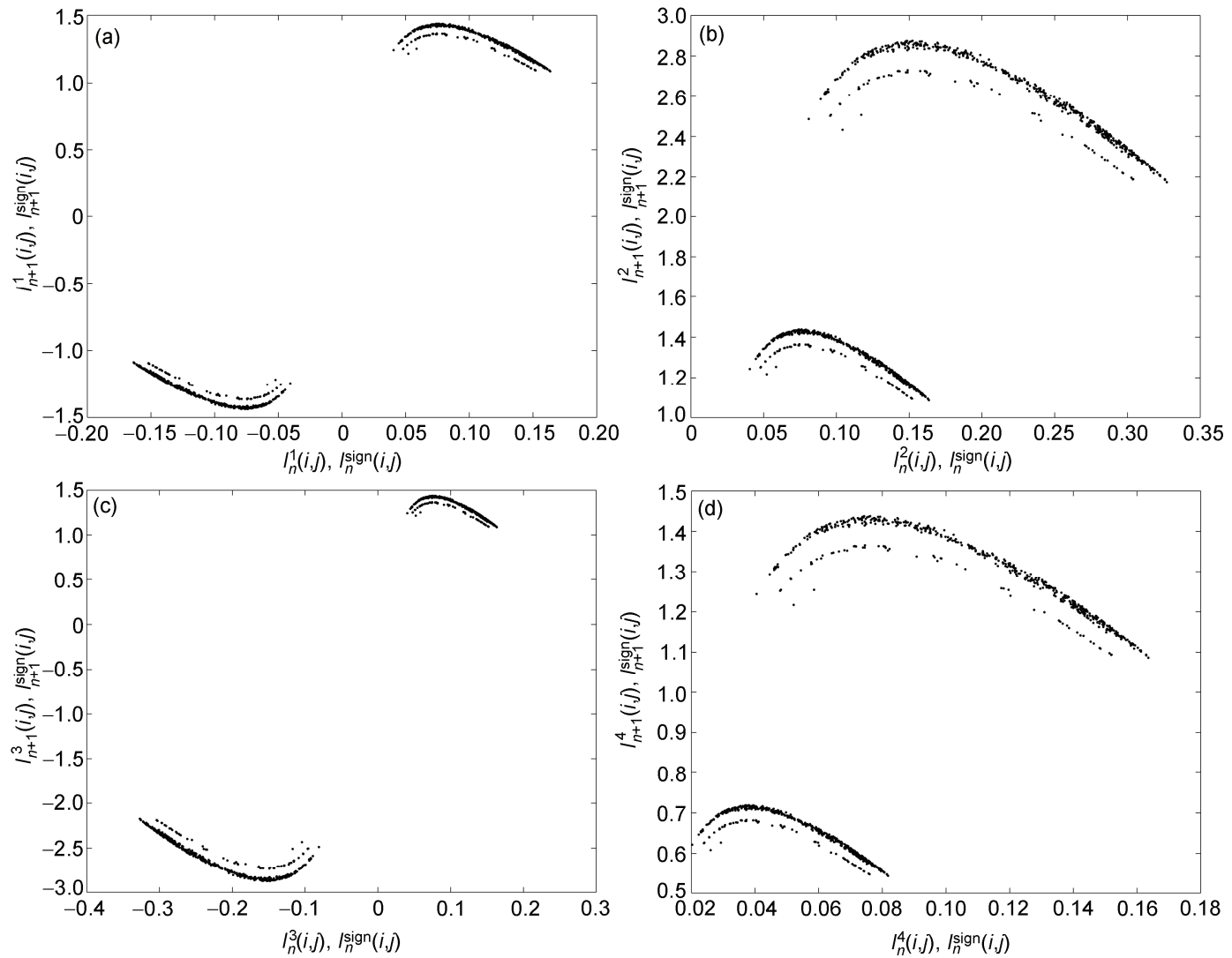

\section{图 9 网络节点与目标信号的相图}

Figure 9 Phase diagrams of the network node and the target signal.

光网络与目标信号实现同步比例传输后 $e_{n}^{k}(i, j)=0$. 同时, 基于(7)式可知 $I_{n}^{k}(i, j)=\beta_{k} I_{n}^{\mathrm{sign}}(i, j)$. 比例传输 因子 $\beta_{k}$ 分别取 $0.5,2$ 以及 -0.5 , 意味着网络 3 个节点 的相图分别是目标信号相图的 0.5 倍、 2 倍和 -0.5 倍, 这一结论与图 7 的情形相对应. 上述仿真结果表明, 激光网络的各节点均按照比例传输因子的大小同步 传输了目标信号.

对应图 5(b)的网络连接, 保持网络其他参量数值 不变, 比例传输因子 $\beta_{k}$ 任取为 $\beta_{1}=-1, \beta_{2}=2, \beta_{3}=-2$, $\beta_{4}=0.5$. 仿真模拟 $n=500$ 时激光网络节点状态变量与 目标信号之间误差的时空演化以及网络节点与目标 信号的相图如图 8 和 9 所示. 我们发现网络对目标信 号的同步比例传输性能依然十分稳定.

我们同样仿真了图 5(c)所示的网络连接甚至更 多节点、任意连接的网络对目标信号的同步比例传输
性能. 同样得到了稳定的传输效果. 这里, 仿真图就 不重复展示.

\section{5 结论}

我们研究了环形腔激光器构成的激光网络对信号 的同步比例传输问题. 首先介绍了环形腔激光器光强 的输出特性以及激光网络的构成. 进一步依据稳定性 理论设计了同步比例传输技术用以实现激光网络对目 标信号的同步比例传输. 仿真结果表明, 当调节参量 $\xi_{k}$ 和配置参量 $\mu_{k}$ 满足 $0<\mu_{k}<2$ 和 $-2<\xi_{k}<0$ 时, 不论比例传输 因子 $\beta_{k}$ 取何值, 该激光网络均可以按照比例传输因子 的大小同步传输目标信号. 而且网络节点之间的连接 方式以及节点数目对整个网络同步比例传输的稳定性 没有影响, 说明该同步比例传输技术是普适和有效的. 


\section{参考文献}

1 Lugiato L A, Milani M. Disappearance of laser instabilities in a Gaussian cavity mode. Opt Commun, 1983, 46: 57-60

2 Pecora L M, Carroll T L. Master stability functions for synchronized coupled systems. Phys Rev Lett, 1998, 80: 2109-2112

3 Gade P M, Hu C K. Synchronous chaos in coupled map lattices with small-world interactions. Phys Rev E, 2000, 62: 6409-6413

4 Earl M G, Strogatz S H. Synchronization in oscillator networks with delayed coupling: A stability criterion. Phys Rev E, 2003, 67: 036204

5 Lü J H, Yu X H, Chen G R. Chaos synchronization of general complex dynamical networks. Physica A, 2004, 334: 281-302

6 Lü J H, Chen G R. A time-varying complex dynamical network model and its controlled synchronization criteria. IEEE Trans Automat Cont, 2005, 50: 841-846

7 Li P, Yi Z. Synchronization analysis of delayed complex networks with time-varying couplings. Physica A, 2008, 387: $3729-3737$

8 Chen M. Synchronization in time-varying networks: A matrix measure approach. Phys Rev E, 2007, 76: 016104

9 Ji D H, Park J H, Yoo W J, et al. Synchronization criterion for Lur'e type complex dynamical networks with time-varying delay. Phys Lett A, 2010, 374: 1218-1227

10 Zhang H H, Wang Q Y, He X Y, et al. Synchronization stability and firing transitions in two types of class I neuronal networks with short-term plasticity. Neural Networks, 2014, 49: 107-117

11 Szmoski R M, Pereira R F, de Souza Pinto S E. Effective dynamics for chaos synchronization in networks with time-varying topology. Nonlinear Sci Numer Simulat, 2013, 18: 1491-1498

12 Li N, Cao J D. New synchronization criteria for memristor-based networks: Adaptive control and feedback control schemes. Neural Networks, 2015, 61: 1-9

13 Bhowmick S K, Amritkar R E, Dana S K. Experimental evidence of synchronization of time-varying dynamical network. Chaos, 2012, 22: 023105

14 Yanagita T. Design of oscillator networks with enhanced synchronization tolerance against noise. Phys Rev E, 2012, 85: 056206

15 Selivanov A A, Lehnert J, Dahms T, et al. Adaptive synchronization in delay-coupled networks of Stuart-Landau oscillators. Phys Rev E, 2012, 85: 016201

16 Watanabe T. Rich-club network topology to minimize synchronization cost due to phase difference among frequency-synchronized oscillators. Physica A, 2013, 392: 1246-1255

17 Du H Y. Adaptive open-plus-closed-loop method of projective synchronization in drive-response dynamical networks. Commun Nonlinear Sci Numer Simulat, 2012 17: 3353-3359

18 Jeong S C, Ji D N, Park J H, et al. Adaptive synchronization for uncertain chaotic neural networks with mixed time delays using fuzzy disturbance observer. Appl Math Comput, 2013, 219: 5984-5995

19 Yang X S, Cao J D. Hybrid adaptive and impulsive synchronization of uncertain complex networks with delays and general uncertain perturbations. Appl Math Comput, 2014, 227: 480-493

20 Wu Y Q, Li C P, Yang A L, et al. Pinning adaptive anti-synchronization between two general complex dynamical networks with nondelayed and delayed coupling. Appl Math Comput, 2012, 218: 7445-7452

$21 \mathrm{Wu} \mathrm{X} \mathrm{J,} \mathrm{Lu} \mathrm{H} \mathrm{T.} \mathrm{Hybrid} \mathrm{synchronization} \mathrm{of} \mathrm{the} \mathrm{general} \mathrm{delayed} \mathrm{and} \mathrm{non-delayed} \mathrm{complex} \mathrm{dynamical} \mathrm{networks} \mathrm{via} \mathrm{pinning} \mathrm{control.}$ Neurocomputing, 2012, 89: 168-177

22 Skardal P S, Taylor D, Sun J, et al. Erosion of synchronization in networks of coupled oscillators. Phys Rev E, 2015, 91 : 010802

23 Zhang R, Yang Y Q, Xu Z Y, et al. Function projective synchronization in drive-response dynamical network. Phys Lett A, 2010, 374: 3025-3028

24 Bao H B, Cao J D. Projective synchronization of fractional-order memristor-based neural networks. Neural Networks, 2015, 63: 1-9

25 Li Z, Xue X. Outer synchronization of coupled networks using arbitrary coupling strength. Chaos, 2010, 20: 023106

26 Wang J W, Ma Q H, Zeng L, et al. Mixed outer synchronization of coupled complex networks with time-varying coupling delay. Chaos, 2011, 21: 013121

27 Du H Y. Adaptive open-plus-closed-loop method of projective synchronization in drive-response dynamical networks. Commun Nonlinear Sci Numer Simulat, 2012 17: 3353-3359

28 Yang L X, Jiang J. Adaptive synchronization of drive-response fractional-order complex dynamical networks with uncertain parameters. Nonlinear Sci Numer Simulat, 2014, 19: 1496-1506

29 谭维翰, 陆伟平. 激光振荡输出的分岔与混沌. 科学通报, 1988, 33: 17-21

30 Wang P Y, Xie P, Dai J H, et al. Stabilization, Selection, and tracking of unstable patterns by weak spatial perturbations. Phys Rev Lett, 1998, 80: 4669-4672 


\title{
Signal proportion transmission with a network constituted by lasers with ring cavities
}

\author{
LV Ling*, LI ChengRen, LI Gang, TIAN Jing, GU JiaJia \& WANG ZhouYang \\ School of Physics and Electronic Technology, Liaoning Normal University, Dalian 116029, China
}

\begin{abstract}
On the basis of stability theory, a synchronization proportion transmission technique is designed to realize the signal proportion transmission of a laser network with spatiotemporal chaotic behavior. Through selecting specific Lyapunov functions, the structure of network node control input is determined. In the synchronization process, the target signal can be input in any node of the network. Using this technology, not only can the laser network transfer synchronously the target signal according to the magnitude of the proportion factor, but also the connection of the network and the number of network nodes are not restricted by any conditions. The simulation results show that the synchronization performance of the network is very stable.
\end{abstract}

synchronization proportion transmission, complex network, spatiotemporal chaos, laser with ring cavity

PACS: 05.45.Xt, 05.45.Pq, 05.45.Ra

doi: 10.1360/SSPMA2015-00087 